\title{
Onicomicose: estudo clínico, epidemiológico e micológico no município de São José do Rio Preto
}

\author{
Onychomycosis: clinical, epidemiological and mycological study \\ in the municipality of São José do Rio Preto
}

\author{
Edna Alves Martins ${ }^{1}$, Letícia Vieira Guerrer ${ }^{1}$, Keith Cássia Cunha ${ }^{1}$, \\ Márcia Maria Costa Nunes Soares ${ }^{2}$ e Margarete Teresa Gottardo de Almeida ${ }^{1}$
}

\begin{abstract}
RESUMO
Estudo clínico-epidemiológico e micológico em 184 pacientes de Hospital Escola: 200 amostras, 142 positivas, 98 leveduras e 68 fungos filamentosos. Candida parapsilosis (47\%) $e$ Trichophyton rubrum (38\%) foram prevalentes. Ao cetoconazol, $100 \%$ de sensibilidade e a anfotericina B, 99\%. Prevalência em mulheres (80\%), adultos (62\%) em pododáctilos (84\%).
\end{abstract}

Palavras-chaves: Onicomicose. Candida parapsilosis. Trichophyton rubrum.

\section{ABSTRACT}

This was a clinical-epidemiological and mycological study on 184 patients at the university hospital: 200 samples, 142 positive samples, 98 yeasts and 68 filamentous fungi. Candida parapsilosis (47\%) and Trichophyton rubrum (38\%) were prevalent. They were 100\% sensitive to ketoconazole and $99 \%$ sensitive to amphotericin B. The highest prevalences were among women (80\%), adults (62\%) and toes (84\%).

Key-words: Onychomycosis. Candida parapsilosis. Trichophyton rubrum.

A onicomicose é a infecção nas unhas determinada por diversas espécies de fungos: dermatófitos, não dermatófitos e leveduras $^{59}$. A distribuição destes diferentes patógenos não é uniforme, e depende de vários fatores tais como clima, área geográfica e migração. Os agentes.

causais das onicomicoses dermatofíticas são os fungos do gênero Trichophyton e Epidermophyton, e excepcionalmente Microsporum. Nos últimos anos, os casos de onicomicoses não dermatofítica, aumentaram rapidamente, sobretudo na Europa, onde são responsáveis por percentagem que varia de 1,6 a 6\%33.

Os fatores que contribuem para a instalação de onicomicose podem ser divididos em: gênero, perturbações circulatórias periféricas, resistência diminuída, traumatismo e fatores de manutenção, como profissão, clima, disfunção hormonal' ${ }^{1}$.

Tendo em vista a alta ocorrência de onicomicose no País, e a ausência de estudos na Cidade de São José do Rio Preto, o presente trabalho teve por objetivo o estudo clínico, epidemiológico e micológico de casos suspeitos.
Foram estudados 200 fragmentos de unha de 184 pacientes com suspeita clínica de onicomicose, atendidos pelo Serviço de Dermatologia do Ambulatório Regional de Especialidades de São José do Rio Preto. A pesquisa foi realizada no Laboratório de Microbiologia da Faculdade de Medicina de São José do Rio Preto (FAMERP), durante o período de 12 meses (julho/2005 a julho/2006). As amostras foram colhidas mediante a raspagem subungueal e/ou superficial da unha, sendo divididas em duas partes: exame direto e cultura utilizando agar Sabouraud dextrose (VETEC), Mycobiotic (DIFCO), Chromagar Candida, auxanograma e zimograma, além de microcultivo em ágar fubá e/ou ágar batata. Avaliou-se o perfil de sensibilidade antifúngica frente às seguintes drogas: anfotericina B, fluconazol, itraconazol e cetoconazol, através do método de difusão em disco proposto pelo National Committee for Clinical Laboratory Standards (NCCLS) ${ }^{11}$, de acordo com o documento M44 P.

Considerando os dados epidemiológicos do estudo, observou-se que dos 184 pacientes estudados, 147 (80\%) eram mulheres. A faixa etária mais acometida foi entre 36 e 64 anos,

\footnotetext{
1. Laboratório de Microbiologia da Faculdade de Medicina de São José do Rio Preto, SP. 2. Curso de Especialização em Enfermagem na Unidade de Terapia Intensiva da Faculdade de Medicina de São José do Rio Preto, SP.

Apoio financeiro: Conselho Nacional de Desenvolvimento Científico e Tecnológico CNPq/PIBIC/ BAP FAMERP

Endereço para correspondência: Prof ${ }^{\mathfrak{a}}$ Margarete Teresa Gottardo Almeida. Lab. de Microbiologia/FAMERP. Av. Brigadeiro Faria Lima 5416, 15090-000 São José do Rio Preto, SP.

Tel: 5517 3201-5741; Fax: 5517 3229-1777

Recebido para publicação em: 26/02/2006

Aceito em: 21/08/2007
} 
representando $62 \%$ dos pacientes estudados, $19 \%$ tinham idade entre 19 e 35 anos, enquanto 17,4\% eram idosos acima de 65 anos, demonstrando ser essa doença de prevalência em adultos. Em $84 \%$ dos casos, a unha dos pododáctilos correspondeu à área mais afetada, e Candida parapsilosis foi mais prevalente em distrofia total e/ou área distal lateral enquanto que Candida albicans, segundo agente mais comum, afetou principalmente o leito ungueal e matriz proximal. Em quirodáctilos, Candida parapsilosis e Candida albicans originaram-se de acometimento distal lateral e distrofia total. Doenças vasculares periféricas e imunodepressão ocorreram em concomitância com doença fúngica em 13,6\% dos pacientes. Quanto à exposição a produtos químicos, 55,4\% faziam uso em atividades profissionais e/ou trabalho doméstico.

A partir das 200 amostras clínicas, obtidas do raspado ungueal, das mãos e dos pés, 142 (71\%) foram positivas para fungo filamentoso e/ou leveduriforme. Destes, 98 correspondeu à leveduras, como isolamento único (69), ou em associação com leveduras (5) e fungo filamentoso (24).

0 isolamento de fungo filamentoso ocorreu em 68 (47\%) amostras e em apenas uma amostra, duas espécies filamentosas. A distribuição das espécies de leveduras e fungos filamentosos é apresentada na Tabela 1.

A análise de suscetibilidade antifúngica para as leveduras mostrou que $100 \%$ das linhagens foram sensíveis ao cetoconazol e 99\% à anfotericina B e mais de 70\% sensíveis ao fluconazol (Figura 1), enquanto 53\% o foram ao itraconazol.

A levedura Candida albicans ${ }^{13}$ e o fungo filamentoso Trichophyton rubrum são os principais agentes causadores de onicomicose $\mathrm{e}^{10}$. Neste estudo, igualmente ao de Djeridane e cols $(2006)^{4}$, houve alta prevalência de Candida parapsilosis (47\%), diferindo dos dados da literatura atual, porém a presença do dermatófito Trichophyton rubrum (38\%) corroborou com os mesmos. A ocorrência de fungos oportunistas como Fusarium sp foi de $14,7 \%$, conhecido como fitopatógeno, tem sido encontrado como agente etiológico emergente, especialmente nas infecções em humanos ${ }^{3}$.

No presente, a maior parte dos pacientes, como adultos (62\%) e mulheres (80\%), corrobora com estudo de Calado e cols $^{3}$, em 2006. Nesses grupos a profissão a utilização de fômites contaminados $^{8}$, traumas por atividade doméstica, exposição a produto químico, são fatores de risco ${ }^{38}$.
As unhas dos pododáctilos foram às áreas mais (84\%) acometidas. 0 uso de calçados fechados e até o comprometimento vascular em membro inferior são considerados fatores primordiais predisponentes para o desenvolvimento de tal infecção ${ }^{2}$.

Pode-se observar que freqüência de sensibilidade ao cetoconazol e a anfotericina $\mathrm{B}$ foi alta. Embora válida como opção terapêutica, estes fármacos não são recomendados pelos dermatologistas, visto que seus efeitos colaterais de toxicidade são altos ${ }^{9}$. Nessa investigação, a atividade in vitro do fluconazol foi expressiva (75\%). Tal fármaco é apresentado com alta atividade contra fungos dermatófitos ${ }^{6}$ e em onicomicose, tem sido considerado como tratamento eficaz, via sistêmica ou tópica, com índices elevados de cura ${ }^{6}$. De modo semelhante, o itraconazol, também é apresentado por vários estudos como método seguro e eficaz ${ }^{1714}$, e na nesta investigação 93\% das amostras foram sensíveis, evidenciando-se a taxa de sensibilidade dose dependente elevada (40\%). Essa tendência à resistência, também detectada por Pappas e cols ${ }^{12}$, em 2004, pode ser devida ao uso indiscriminado da droga, à profilaxia e à não aderência ao esquema terapêutico pelo paciente, fato que justifica eventual falha terapêutica.

0 conhecimento da etiologia da onicomicose fornecida pelo presente estudo, na região de São José do Rio Preto bem

Tabela 1 - Distribuição das espécies de fungos nos isolados clínicos.

\begin{tabular}{|c|c|c|c|}
\hline \multicolumn{2}{|l|}{ Leveduras } & \multicolumn{2}{|c|}{ Filamentosos } \\
\hline espécie & $\begin{array}{c}\text { ocorrência } \\
\%\end{array}$ & espécie & $\begin{array}{c}\text { ocorrência } \\
\%\end{array}$ \\
\hline Candida parapsilosis & 47,0 & Trichophyton rubrum & 38,0 \\
\hline Candida albicans & 20,0 & Fusarium sp & 14,0 \\
\hline Candida guilliermondii & 9,0 & Cladosporium sp & 14,0 \\
\hline Trichosporon beigelli & 8,0 & Scytalidium dimitiatum & 14,0 \\
\hline Candida tropicalis & 7,0 & Aspergillus sp & 7,0 \\
\hline Candida krusei & 1,2 & Epicoccum $\mathrm{sp}$ & 2,0 \\
\hline Candida zeilanoides & 1,2 & Penicillium sp & 2,0 \\
\hline Phaeococcus sp & 1,2 & Epidermophyton floccosum & 2,0 \\
\hline Rhodotorula glutinis & 1,2 & Acremonium sp & 2,0 \\
\hline Sacharomyces cerevisae & 1,2 & Aureobasidium pullulans & 2,0 \\
\hline Trichosporon pullulans & 1,2 & Paecylomyces sp & 2,0 \\
\hline Trichosporon sp & 1,2 & & \\
\hline Hansenula anamala & 1,2 & & \\
\hline Phototheca wickerhamii & 1,0 & & \\
\hline
\end{tabular}

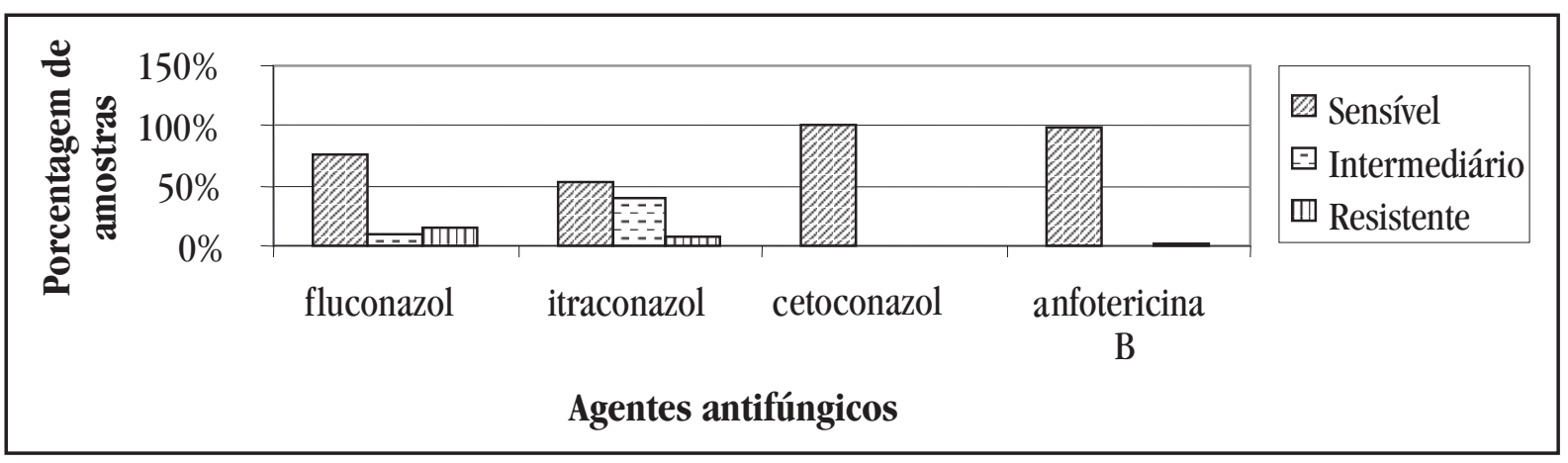

Figura 1-Perfil de sensibilidade das leveduras aos agentes antifúngicos. 
como, a análise dos padrões de sensibilidade a antifúngicos, não estabelecidos anteriormente, permitirão constituir medidas de controle dessa infecção, diminuindo a recidivas e otimização no tratamento.

\section{AGRADECIMENTO}

À Luceli Ferreira de Souza, técnica do Laboratório de Microbiologia da Faculdade de Medicina de São José do Rio Preto, pela colaboração técnica dispensada.

\section{REFERÊNCIAS}

1. Avner S, Nir N, Baruch K, Henri T. Two novel itraconazole pulse therapies for onychomycosis: a 2-year follow-up. The Journal of Dermatological Treatment 7: 117-120, 2006

2. Boonchai W, Kulthanan K, Maungprasat C, Suthipinittham P. Clinical characteristics and mycology of onychomycosis in autoimmune patients. Journal of the Medical Association of Thailand 86: 995-1000, 2003.

3. Calado NB, Souza-Jr F, Gomes NO, Cardoso FR, Zaror LC, Milan EP. Fusarium nail and skin infection: A report of eight cases from Natal, Brazil. Mycopathologia 161: 27-31, 2006

4. Djeridane A, Djeridane Y, Ammar-Khodja A. Epidemiological and aetiological study on tinea pedis and onychomycosis in Algeria. Mycosis 49: 190-196, 2006.
5. Elewski BE. Onychomycosis. Pathogenesis, diagnosis and management. Clinical Micorbiology Review 11: 415-429, 1998.

6. El-Komy MHM. Nailfold fluconazole fluid injection for fingernail onychomycosis Clinical and Experimental Dermatology 31: 465-467, 2006.

7. Gupta AK, Gover MD, Lynde CW. Pulse itraconazole vs. continuous terbinafine for the treatment of dermatophyte toenail onychomycosis in patients with diabetes mellitus. Journal of the European Academy of Dermatology and Venereology 20: 1188-1193, 2006

8. Gupta AK, Ryner JE, Summerbell RC. Onychomycosis: classification and diagnosis. Journal of Drugs in Dermatology 3:51-56, 2004.

9. Janssen Pharmaceutica. Ketoconazole package insert. Janssen Pharmaceutica, Titusville, NJ. 1997.

10. Lacaz CS, Porto E, Martins JEC, Vaccari EMH, Melo NT. Tratado de Micologia Médica Lacaz, 9ª edição, Editora Sarvier, São Paulo, p. 810-835, 2002.

11. National Committee for Clinical Laboratory Standards. Method for antifungal disk diffusion susceptibility testing of yeasts: approved guideline M44-A. Wayne, National Committee for Clinical Laboratory Standards, Wayne, PA, 2004.

12. Pappas PG, Rex JH, Sobel JD, Filler SG, Dismukes WE, Walsh TJ, Edward JE. Diretrizes para o Tratamento de Candidíase. Clinical Infections Disease 38: 161-189, 2004

13. Pontes ZB, Lima EO, Oliveira NM, Dos Santos JP, Ramos AL, Carvalho MF Onycomycosis in João Pessoa city, Brazil. Revista Argentina de Microbiología 34: $95-99,2002$

14. Santos DA, Hamdan JS. In vitro antifungal oral drug and drug-combination activity against onychomycosis causative dermatophytes. Medical Mycology 44: 357-362, 2006

15. Sikder AU, Mamun SA, Chowdhury AH, Khan RM, Hoque MM. Study of oral itraconazole and terbinafine pulse therapy in onychomycosis. Mymensingh Medical Journal 15: 71-80, 2006 